\title{
Costo de las neumonías asociadas a la ventilación mecánica en pacientes adultos en un hospital general en Chile
}

\author{
Elena Véliz y Alberto Fica
}

\section{Cost evaluation of ventilator-associated pneumonia in adult patients in a general hospital in Chile}

Background: Ventilator-associated pneumonia (VAP) is an adverse event that increases morbidity, mortality and costs due to a prolonged stay and requirement of microbiological studies and antimicrobial therapy. There is not recent data of VAP costs in Chile. Aim: To evaluate additional costs in adult patients with VAP compared to controls in a general hospital in the Metropolitan Area. Patients and Methods: Use of the PAHO paired casecontrol protocol for cost evaluation associated to nosocomial infections and estimation of cost in excess per VAP event. Length of stay (LOS) in excess, antimicrobial consumption in daily-defined doses (DDD), and number of microbiological studies were compared between both groups. Results: From 2012 to 2015, 18 patients with VAP events were identified with their respective controls. LOS exceeded 6.1 days on average among patients with VAP respect to controls $(\mathrm{p}<0.05)$. DDD was higher among patients with VAP (difference 11.7 DDD) as well as number of cultures (3.2 higher on average, $\mathrm{p}<0.05)$. Cost in excess per VAP event reached 4,475 USD. Conclusions: In our Centre, VAP events are associated to a higher LOS, antimicrobial consumption and microbiological studies.

Key words: Ventilator-associated pneumonia, cost analysis, adults, Pan American Health Organization.

Palabras clave: Neumonía asociada a la ventilación mecánica, análisis de costo, adultos, Organización Panamericana de la Salud.

\section{Introducción}

L

as infecciones asociadas a la atención de la salud (IAAS) corresponden a eventos adversos que aumentan la morbi-mortalidad y los costos. De ellas, las neumonías asociadas a la ventilación mecánica (NAVM) presentan una gran variabilidad de costos y un alto impacto en mortalidad. En E.U.A. se estima que al año, 5 a 20\% de los pacientes con ventilación mecánica invasora presentan NAVM en unidades críticas ${ }^{1}$.

Los costos aproximados por esta infección van desde 1.728 USD a 10.000 USD por evento ${ }^{2,3}$. En Canadá se ha estimado un costo de 46 millones de dólares por el total de NAVM anuales ${ }^{4}$. El aumento en los gastos hospitalarios está dado por los días adicionales de internación y los procedimientos diagnósticos y terapéuticos para su tratamiento, entre ellos, cultivos y uso de antimicrobianos $^{5-7}$. Los excesos en días cama hospitalaria, van desde 7 a 30 días $^{8}$.

Del total de las IAAS, la NAVM presenta mayor letalidad general, con cifras que van de 5,8 a $50 \% \%^{9,10}$. Además, la NAVM constituye un factor de riesgo independiente de mortalidad (OR 2,1; IC: 95\% 1,2-3,6) 3 ,4,7 con una mortalidad atribuible de 35 a $41 \% 0^{4,8,9,11,12}$.

En Chile, de acuerdo al último reporte de vigilancia epidemiológica nacional, se estima que anualmente se producen cerca de 1.600 NAVM entre pacientes adultos y pediátricos, siendo de mayor prevalencia en adultos. Este evento ocupa el $6^{\circ}$ lugar en la frecuencia de IAAS ${ }^{13}$.

Un análisis de costo realizado en varios hospitales chilenos, durante el año 2002, reveló que las NAVM se asocian a un exceso de estadía hospitalaria de 8,1 a 44,7 días y de 28 a 73 dosis diaria definida (DDD) en antimicrobianos, cifras similares a las aportadas por Nercelles y cols., en un trabajo posterior ${ }^{6,14}$.

La información sobre costos asociados a NAVM en hospitales nacionales es escasa y proveniente de estudios realizados hace más de una década, siendo necesaria una actualización al respecto, especialmente si se considera que los costos pueden haberse reducido por tratamientos más eficaces o menores costos en antimicrobianos o insumos, considerando las diferentes formas en que se gestionan los gastos en los centros de salud privados, institucionales y públicos ${ }^{15}$. El contar con información de costos y uso de recursos hospitalarios es de gran utilidad para estimar el beneficio de los programas de intervención en prevención de IAAS y su impacto en términos económicos. Estos antecedentes determinaron la necesidad de efectuar un estudio cuyo objetivo fue cuantificar el costo de las NAVM en un hospital general.
Hospital Militar de Santiago. Unidad de Infecciones Asociadas a la Atención de Salud (EV, AF).

AF se desempeña actualmente en Hospital Base Valdivia.

Conflicto de interés: ninguno. Financiamiento: ninguno.

Recibido: 22 de noviembre de 2016

Aceptado: 20 de septiembre de 2017

Correspondencia a: Elena Véliz eveliz@hms.cl 


\section{Pacientes y Método}

Este estudio se realizó en un hospital de alta complejidad de la Región Metropolitana de Chile, utilizando la metodología caso-control de evaluación de costo de las infecciones intrahospitalarias propuesta por la Organización Panamericana de la Salud (OPS) ${ }^{2}$.

Se recolectaron los casos de NAVM de UCI adulto, según los datos obtenidos de la vigilancia epidemiológica de la Unidad de Control de Infecciones del establecimiento, entre los años 2012 y 2015. Los criterios de selección de NAVM correspondieron a las definiciones establecidas por el Consenso Chileno de NAVM y que considera la aparición de nuevos infiltrados pulmonares que no se modifican con kinesiterapia respiratoria, asociados a aparición de expectoración purulenta o cambios en las características de la expectoración, con un cultivo cuantitativo de un agente patógeno obtenido de la vía aérea sobre el valor de corte $\left(\geq 10^{6} \mathrm{ufc} / \mathrm{mL}\right.$ para aspirado endotraqueal $\mathrm{y} \geq 10^{4} \mathrm{ufc} / \mathrm{mL}$ para lavado bronco-alveolar $)^{16}$.

De acuerdo a estos criterios, se detectaron 18 casos de NAVM en el período de estudio, correspondientes al servicio de UCI adultos. Este servicio es parte de un hospital de alta complejidad (nivel terciario), donde se atiende mayoritariamente a personal de las FF.AA. y sus familiares, pacientes "Ley de Urgencia" y público en general, incluyendo pacientes pertenecientes al seguro público de salud (FONASA). La Unidad de Cuidados Intensivos cuenta con nueve camas y tiene un índice ocupacional que varía entre 55 y $60 \%$, incluyendo pacientes con patología médica, pacientes post-cirugía y neuro-críticos. Los pacientes con quemaduras graves son derivados a centros especializados para su manejo multidisciplinario. Esta Unidad cuenta con un total anual de egresos de 288 pacientes y con un total de 1.758 días cama ocupada (período 2016) y gran parte de la población está compuesta por adultos mayores. Se han observado bajas tasas de NAVM ( 3,3 a 6,3 por 1.000 días de VM) con respecto a la referencia nacional $(14,8$ por 1.000 días de VM, según indicadores MINSAL 2015) y que en promedio corresponden a 4 a 5 casos al año.
Se revisó la ficha de cada paciente registrando las variables: edad, sexo, diagnóstico, cultivos y antimicrobianos utilizados, de acuerdo a lo solicitado en formulario OPS. Los controles se obtuvieron en forma aleatoria para cada caso, pareados por género, y dentro de los pacientes que habían requerido ventilación mecánica en UCI durante el mismo mes pero que no presentaron neumonía. El pareo por edad consideró \pm 10 años. En las situaciones en que había más de un paciente coincidente con la edad del caso, se les asignó un número correlativo y se les randomizó a través de randomizer.org (https://www.randomizer.org) ${ }^{17}$.

Se seleccionaron como indicadores económicos el exceso de días de hospitalización, exceso de uso de antimicrobianos en dosis diaria definida (DDD) y el exceso de cultivos entre casos y controles.

Los costos fueron obtenidos de la Dirección Comercial del establecimiento. Los excesos de costo se analizaron en moneda local (pesos chilenos), valores que fueron convertidos a USD, considerando la paridad cambiaria a enero de 20161 ( 1 USD = 711 CLP).

Las variables continuas se compararon mediante pruebas no paramétricas (prueba U de Mann-Whitney) considerando un valor significativo $\mathrm{p}<0,05$.

El estudio fue aprobado por el Comité de Ética del establecimiento.

\section{Resultados}

Se evaluaron 18 casos, con sus respectivos controles con un nivel de pareo, de $100 \%$ respecto a la variable medida.

Al analizar los costos por cada indicador económico se observa lo siguiente:

- La estadía hospitalaria promedio en pacientes con NAVM fue de 31 días y para el grupo control fue de 24,9 días, estableciendo una diferencia de casi una semana (6,1 días) (Tabla 1).

- Los pacientes con NAVM tuvieron una prescripción de antimicrobianos mayor que los controles, con una diferencia promedio de 11,7 DDD (Tabla 1).

Tabla 1. Comparación de estadía hospitalaria, consumo de antimicrobianos y número de cultivos entre pacientes con NAVM* y grupo control. Hospital Militar de Santiago, 2012-2015

\begin{tabular}{lccc}
\hline Parámetro & $\begin{array}{c}\text { Grupo NAVM } \\
\mathbf{n}=\mathbf{1 8}\end{array}$ & $\begin{array}{c}\text { Grupo control } \\
\mathbf{n}=\mathbf{1 8}\end{array}$ & Diferencia \\
Estadía hospitalaria en días totales & 558 & 449 & $109 * *$ \\
Estadía hospitalaria en días, valor promedio & 31,0 & 24,9 & 3,1 \\
Consumo de antimicrobianos DDD, valor promedio & 48,3 & 36,6 & 3,0 \\
Número de cultivos, valor promedio & 6,2 & 11,7 \\
\hline *NAVM: neumonía asociada a la ventilación mecánica. ** Diferencia significativa por prueba no paramétrica de Mann Whitney (p < 0,05).
\end{tabular}




\begin{tabular}{|c|c|c|c|c|c|c|}
\hline Parámetro & $\begin{array}{l}\text { Casos } \\
\text { (a) }\end{array}$ & $\begin{array}{l}\text { Controles } \\
\text { (b) }\end{array}$ & $\begin{array}{l}\text { Diferencia } \\
\quad(a-b)\end{array}$ & $\begin{array}{l}\text { Costo día cama en } \\
\text { pesos chilenos }\end{array}$ & $\begin{array}{l}\text { Diferencia de costo } \\
\text { en pesos chilenos }\end{array}$ & $\begin{array}{c}\text { Valor en } \\
\text { USD** }\end{array}$ \\
\hline \multicolumn{7}{|l|}{ Días de hospitalización } \\
\hline Sala (Medicina-Cirugía) & 203 & 202 & 1 & 95.821 & 95.821 & 135 \\
\hline UPC & 355 & 247 & 108 & 464.298 & 50.144 .184 & 70.526 \\
\hline Total por hospitalización & 558 & 449 & 109 & & 50.240 .005 & 70.661 \\
\hline \multicolumn{7}{|l|}{ Consumo de antimicrobianos } \\
\hline DDD & 869,6 & 659,4 & 210,2 & & 6.795 .587 & 9.557 \\
\hline \multicolumn{7}{|l|}{ Cultivos } \\
\hline Hemocultivos & 51 & 33 & 18 & & 160.380 & 226 \\
\hline Urocultivos & 22 & 12 & 10 & & 43.296 & 61 \\
\hline Cultivos de vía aérea & 17 & 6 & 11 & & 18.000 & 25 \\
\hline Otros cultivos & 21 & 2 & 19 & & 8.892 & 13 \\
\hline Total de cultivos & 111 & 53 & 58 & & 230.568 & 324 \\
\hline Total (hospitalización, consumo y cultivos) & & & & & 57.266 .160 & 80.542 \\
\hline Por caso & & & & & 3.181 .453 & 4.475 \\
\hline
\end{tabular}

- También se observó un exceso de solicitud de cultivos en los casos respecto de los controles, con una diferencia promedio de 3,2 .

- Las diferencias para estadía hospitalaria y cultivos fueron significativas $(\mathrm{p}<0,05)$.

El cálculo de los costos por hospitalización, considerando la contribución de los días en salas generales y en unidades críticas o semi-críticas, demostró un valor en exceso superior a 57 millones de pesos, equivalente a USD 80.542 (Tabla 2). Esta cifra representó 87,7\% del exceso de costos calculados con este protocolo.

Para el uso de antimicrobianos, la diferencia superó los 6 millones de pesos (9.557 USD, Tabla 2) y contribuyó con $11,8 \%$ al exceso de costos.

En contraste, el exceso de cultivos contribuyó sólo con $0,5 \%$ al exceso de gastos (cerca de 230.000 pesos) (Tabla 2).

El exceso de gasto por evento de NAVM superó los 3 millones de pesos y fue equivalente a 4.475 USD.

De esta manera, las NAVM acumuladas durante cuatro años (2012-2015) representaron para el establecimiento un costo asociado de 57 millones de pesos, equivalente a 80 mil dólares.

El análisis del consumo de antimicrobianos indicó una tendencia a una mayor prescripción y uso de carbapenémicos, colistín, levofloxacina y vancomicina, siendo significativo para el caso de meropenem y colistín $(\mathrm{p}<0,05)$ (Tabla 3 para algunos antimicrobianos seleccionados). El exceso de costo por carbapenémicos, colistín y vancomicina fue cercano a 2 millones de pesos (Tabla 3 ).
Tabla 3. Distribución del consumo de antimicrobianos seleccionados en pacientes con NAVM $^{*}$ y grupo control, Hospital Militar de Santiago, 2012-2015

\begin{tabular}{|c|c|c|c|c|c|}
\hline \multirow[t]{2}{*}{ Compuesto } & \multicolumn{3}{|c|}{ Consumo en DDD } & \multirow{2}{*}{$\begin{array}{l}\text { Diferencia en } \\
\text { pesos chilenos }\end{array}$} & \multirow{2}{*}{$\begin{array}{c}\text { Diferencia en } \\
\text { USD** }\end{array}$} \\
\hline & Casos (a) & Controles (b) & $D=a-b$ & & \\
\hline \multicolumn{6}{|l|}{$\beta$-lactámicos } \\
\hline Imipenem & 59,8 & 28 & 31,8 & 179.765 & 252,8 \\
\hline Meropenem & 125 & 47,5 & $77,5^{*}$ & 708.815 & 996,9 \\
\hline \multicolumn{6}{|l|}{ Quinolonas } \\
\hline Levofloxacina & 21,4 & 11,5 & 9,9 & 117.810 & 165,7 \\
\hline \multicolumn{6}{|l|}{ Otros } \\
\hline Colistín & 80 & 0 & $80^{*}$ & 932.960 & $1.312,1$ \\
\hline Cotrimoxazol & 0 & 0 & 0 & 0 & 0 \\
\hline Fluconazol & 25,2 & 19,5 & 5,7 & 20.000 & 28,1 \\
\hline Linezolid & 1 & 0 & 1 & 108.528 & 152,6 \\
\hline Vancomicina & 87,4 & 49,6 & 37,8 & 188.794 & 265,5 \\
\hline Total & 571,4 & 363,4 & 243,7 & 2.256 .672 & $3.173,7$ \\
\hline
\end{tabular}

\section{Discusión}

En este trabajo se observa que las NAVM acumuladas durante cuatro años (2012-2015) representaron para el establecimiento un costo asociado sobre 57 millones de pesos, equivalente a 80 mil dólares. El valor por evento fue superior a los 4.000 USD, siendo éste el primer estudio en Chile que valoriza el costo de las NAVM, ya 
que otros estudios sólo han evaluado el exceso de estadía hospitalaria y de consumo de antimicrobianos ${ }^{6,14}$.

Estudios de costo realizados en E.U.A. en el año 2008 y 2013, muestran cifras 10 veces más altas que lo obtenido en el presente estudio, en rangos que van de 40.144 a 46.000 USD, diferencias explicadas por su mayor estructura de $\operatorname{costos}^{4,18-20}$. La evidencia indica que la atención hospitalaria de los pacientes que presentan NAVM triplica o duplica el costo comparado con aquellos pacientes que no la presentan ${ }^{7,19,21}$ e incluso puede llegar a ser siete veces superior ${ }^{22}$.

En la literatura médica se han descrito cifras variables de costo, tales como 249, 1.728 y 2.050 USD por evento $^{22-27}$ y en otros casos, valores más cercanos a los obtenidos en el presente estudio ${ }^{8}$. Otros estudios realizados en hospitales de nivel terciario en países de Centro y Sudamérica, muestran cifras que van desde 8.000 a 13.000 USD como exceso de costo de $\mathrm{NAVM}^{3,7,28,29}$. Las variaciones en el costo pueden deberse a múltiples factores como el costo del día cama, la cobertura de la terapia empírica inicial, que de ser inadecuada, aumenta la probabilidad de fracaso y mayor estadía hospitalaria, la intensidad del trabajo kinésico que incide en la recuperación y posibles diferencias en la sedación de los pacientes que pueden dificultar la recuperación de ellos. Chastre, en una revisión sistemática plantea que la diversidad de costos en NAVM también se relaciona con el tipo de pacientes en que se produce el evento ya que los pacientes con trauma o que ingresan por cuadros respiratorios tienen un costo de la atención mayor, de 30 a 50\%, comparado con otro tipo de pacientes con $\mathrm{NAVM}^{10}$. Esto resalta la importancia de hacer estudios locales por la gran variabilidad entre países. Comparativamente, además, el costo en exceso de un evento de NAVM fue menor al costo por evento de bacteriemia asociado a catéter venoso central en nuestra misma institución (7.286 USD) $)^{30}$.

Los días de hospitalización concentraron el principal exceso de costo, en una proporción cercana a $90 \%$, similar a lo reportado en la literatura científica ${ }^{6,23,25}$. Sólo en un estudio de costo, de Bolivia, se observa que el exceso de costo por días de hospitalización es sobrepasado por el exceso de uso de antimicrobianos ${ }^{22}$.

El exceso de estadía hospitalaria observado en nuestro estudio equivale casi a una semana adicional, con un valor de 3.900 USD por evento, cifra similar a la encontrado en un hospital terciario de Lima y en E.U.A. ${ }^{3,24}$. Sin embargo, los tiempos de estadía encontrados en la literatura médica son habitualmente mayores, donde duplica y hasta quintuplica la de los controles ${ }^{14,22-25,29}$. Esto, no necesariamente se traduce en un aumento proporcional de los costos, ya que los excesos de estadía de dos a siete semanas no sobrepasan los 3.000 USD en hospitales sudamericanos de Argentina, Perú, Bolivia o Ecuador ${ }^{22-24}$. Lo contrario sucede en E.U.A., donde los costos para dos semanas superan los 90.000 USD $^{31}$. Tal como se observa en el presente estudio, el exceso de costo por estadía hospitalaria está dado por la hospitalización en UCI. Al respecto, el exceso de estadía hospitalaria en UCI fue cuatro veces más en los casos, respecto de los controles, según un estudio de costo realizado en un hospital terciario de E.U.A. De la misma manera, en un estudio de costo realizado en un hospital guatemalteco se observó un exceso de estadía hospitalaria promedio de 10,5 días, donde $50 \%$ correspondió a $\mathrm{UCI}^{27}$. En nuestro centro, la estadía en exceso por NAVM en UCI fue dos veces superior a la de los pacientes que cursan con bacteriemia por catéter ${ }^{30}$ un rasgo similar a lo descrito previamente en 23 instituciones de salud de Chile ${ }^{14}$.

El exceso de costo por concepto de cultivos microbiológicos fue el doble en los casos respecto de los controles, aunque comparativamente sólo representó $1 \%$ del valor total. En el presente estudio se solicitaron en promedio 3,2 exámenes adicionales respecto a los controles, con un costo de 2 USD. Otros estudios hacen referencia a valores que van de 4 a $5 \mathrm{USD}^{24}$, aunque en un estudio en un hospital paraguayo se observa un valor mayor que lo habitual (30 USD) ${ }^{28}$. Así, comparativamente con los otros indicadores económicos, el exceso de costo por este concepto es marginal. El exceso de gastos por estudios microbiológicos en bacteriemia por catéter es variable, con cifras que van desde 3,6 USD a 40 USD y en el caso de infección urinaria asociada a catéter urinario, los costos en exceso van de 2,8 USD a $37 \mathrm{USD}^{27,28,30}$.

Los antimicrobianos fueron indicados más en los casos que en los controles (diferencia promedio > 11 DDD) con un costo adicional de 530 USD por cada caso de NAVM. Esta cifra está dentro del rango descrito en otros estudios en Chile y en países de Centro y Sudamérica (por ejemplo, rango 2,5 a 58 DDD en exceso en Chile $)^{6,14,22,23,25}$. Estudios realizados en hospitales de Paraguay muestran excesos de costo que van desde 644 a 2.000 USD $^{28,32}$.

Este estudio sólo contempló los costos directos, tal como lo hacen otras evaluaciones de este tipo. Sin embargo, es importante considerar los costos indirectos, tales como la mortalidad, que específicamente en NAVM es mayor que en el resto de las IAAS ${ }^{4,8,9,11,33}$. El análisis de mortalidad de nuestros pacientes pudo constatar que 5 de los 18 pacientes afectados fallecieron a causa de esta infección nosocomial $(27,8 \%$ de mortalidad atribuible), cifra esperada dentro del impacto de mortalidad que la NAVM tiene en los pacientes ${ }^{8,10,19}$. Estos costos no son visibles ni medibles pero generan alto impacto psicológico y social.

En conclusión, los resultados de este trabajo revelan que, para nuestro centro, cada NAVM tuvo un costo de 4.425 USD, constituyendo la estadía hospitalaria adicional el factor determinante de este mayor gasto. Los eventos 
de NAVM generaron también un mayor consumo de antimicrobianos y en forma marginal, más estudios microbiológicos.

\section{Resumen}

Introducción: La neumonía asociada a la ventilación mecánica (NAVM) es un evento adverso que aumenta la morbi-mortalidad y costos. Genera días adicionales de hospitalización y de procedimientos diagnósticos y terapéuticos para su tratamiento. No existen datos actualizados nacionales respecto al exceso de costos por NAVM. Objetivo: Dimensionar el costo de las NAVM en un hospital general de la Región Metropolitana. Pacientes y Métodos: Aplicación del protocolo caso-control de costos de infecciones intrahospitalarias de la Organización Panamericana de la Salud (OPS) y cálculo directo de gasto en exceso por evento de NAVM. Se comparó el exceso de días de hospitalización, de antimicrobianos en dosis diaria definida (DDD) y de cultivos. Resultados: Se recolectaron 18 casos de NAVM entre los años 2012 y 2015 en pacientes adultos. Se seleccionaron 18 controles pareados por edad y género. Se observó una mayor estadía promedio de 6,1 días en los casos $(\mathrm{p}<0,05)$, una mayor prescripción de antimicrobianos (diferencia promedio de 11,7 DDD, sin significancia estadística) y un exceso de solicitud de cultivos con una diferencia promedio de 3,2 $(\mathrm{p}<0,05)$. El costo unitario por NAVM fue de 4.475 USD. Conclusiones: Los eventos de NAVM generan una mayor estadía hospitalaria, consumo de recursos diagnósticos y terapéuticos.

\section{Referencias bibliográficas}

1.- Klompas M, Branson R, Eichenwald E, Greene L, Howell M, Lee G, et al. Strategies to prevent ventilator-associated pneumonia in acute care hospitals: 2014 Update. Infect Control 2014; 35: 915-36.

2.- Organización Panamericana de la Salud. Protocolo para determinar el costo de la infección intrahospitalaria. Washington, D.C.: OPS, 1999 (OPS/HCP/HCT/16/00)

3.- Safdar N, Dezfulian C, Collard H R, Saint $\mathrm{S}$. Clinical and economic consequences of ventilator-associated pneumonia: a systematic review. Crit Care Med 2005; 33: 2184-93.

4.- Muscedere J, Martin C, Heyland D. The impact of ventilator-associated pneumonia on the Canadian health care system. Crit Care 2008; 23: 5-10.

5.- Álvarez-Hernández G, Amaro-Ortega C. Attributable costs and risk factors for pediatric nosocomial infection at a Pediatric Hospital of the State of Sonora. Bol Med Hosp Infant Mex 2010: 67; 118-27.

6.- Brenner P, Nercelles P, Pohlenz M. Costo de las infecciones intrahospitalarias en hospitales chilenos de alta y mediana complejidad. Rev Chilena Infectol 2003; 20: 285-90.

7.- Warren D K, Shukla S J, Olsen M A, Kollef M H, Hollenbeak C S, Cox M J, et al. Outcome and attributable cost of ventilator-associated pneumonia among intensive care unit patients in a suburban medical center. Crit Care Med 2003; 31: 1312-7.

8.- Jarvis W R. Selected aspects of the socioeconomic impact of nosocomial infections: morbidity, mortality, cost, and prevention. Infect Control Hosp Epidemiol 1996; 17: 552-7.

9.- Bercault N, Boulain T. Mortality rate attributable to ventilator-associated nosocomial pneumonia in an adult intensive care unit: a prospective case-control study. Crit Care Med 2001; 29: 2303-9.

10.- Chastre J Yves-Fagon J, Pompidou G. Ventilator-associated pneumonia. Am J Respir Crit Care Med 2002: 165: 867-903.

11.- Rosenthal V D, Guzmán S, Orellano P W. Nosocomial infections in medical-surgical intensive care units in Argentina: attributable mortality and length of stay. Am J Infect Control 2003; 31: 291-5.

12.- Plowman R, Graves N, Griffin M A, Roberts J A, Swan A V, Cookson B, et al. The rate and cost of hospital-acquired infections occurring in patients admitted to selected specialties of a district general hospital in England and the national burden imposed. J Hosp Infect 2001; 47: 198-209.

13.- Otaíza F, Orsini M, Pohlenz M. Informe de Vigilancia de Infecciones Asociadas a la Atención en Salud. MINSAL 2014. Disponible en: http://web.minsal.cl/wp-content/ uploads/2016/10/informe-IAAS-2014.pdf (Accedido el 19 de noviembre de 2016).

14.- Nercelles P, Herrera R, Peirano L, Villarroel M. Exceso y estructura de costos de las infecciones intrahospitalarias en un hospital de nivel terciario de Valparaíso, Chile. En: González-Salvatierra R. Ed. Costo de la infección nosocomial en nueve países de América Latina. Organización Panamericana de la Salud. Washington D.C.: OPS, 2003. Pl-15. Disponible en: http://www2.paho.org/ $\mathrm{hq} / \mathrm{dmdocuments} / 2011 /$ eer-amr-costo-infecchospital.pdf (Accedido el 3 de marzo de 2016).

15.- Cid P C, Bastías S G. Evaluation of financial status of public hospitals considering the updated costs of their services. Rev Med Chile 2014; 142: 161-7.

16.- Fica A, Cifuentes M, Hervé B. Actualización del Consenso Neumonía Asociada a Ventilación Mecánica" Primera parte: Aspectos diagnósticos. Rev Chilena Infectol 2011; 28: 130-51.

17.- Randomización. Acceso a través de https:// www.randomizer.org. (Accedido el 19 de noviembre de 2016).

18.- Zimlichman E, Henderson D, Tamir O, Franz C, Song P, Yamin C K, et al. Health careassociated infections: a meta-analysis of costs and financial impact on the US health care system. JAMA Intern Med 2013; 173: 2039-46.

19.- Rello J, Ollendorf D, Oster G, Vera-Llonch M, Bellm L, Redman R, et al. Epidemiology and outcomes of ventilator-associated pneumonia in a large US database. Chest 2002; 122: 2115-21.

20.- Kollef M, Hamilton C, Ernst F. Economic impact of ventilator-associated pneumonia in a large matched cohort. Infect Control Hosp Epidemiol 2012; 33: 250-6.

21.- Alp E, Kalin G, Coskun R, Sungur M, Guven $\mathrm{M}$, Doganay M. Economic burden of ventilatorassociated pneumonia in a developing country. J Hosp Infect 2012; 81: 128-30.

22.- Coleoni M, Tejerina B, Salazar J, Damiani E, Castro R. Costo de las infecciones nosocomiales en tres hospitales de Bolivia. En: González-Salvatierra R. Ed. Costo de la infección nosocomial en nueve países de América Latina. Organización Panamericana de la Salud. Washington D.C.: OPS, 2003. Pl-15. Disponible en: http://www2.paho.org/ hq/dmdocuments/2011/eer-amr-costo-infecchospital.pdf (Accedido el 3 de marzo de 2016).

23.- Rosenthal V D, Guzmán S, Migone O. Costo de las infecciones nosocomiales en dos unidades de cuidados intensivos de un hospital privado de la Argentina. En: González-Salvatierra R. Ed. Costo de la infección nosocomial en nueve países de América Latina. Organización Panamericana de la Salud. Washington D.C.: 
OPS, 2003. P1-15. Disponible en: http://www2. paho.org/hq/dmdocuments/2011/eer-amr-costoinfecc-hospital.pdf (Accedido el 3 de marzo de 2016).

24.- Castilla T, Yagui M, Remenyi M, Tello Y, Valdivieso F, Llanos F, et al. Costo de infecciones asociadas a cesárea y al uso de ventilación mecánica en un hospital de Lima Perú. En: González-Salvatierra R. Ed. Costo de la infección nosocomial en nueve países de América Latina. Organización Panamericana de la Salud. Washington D.C.: OPS, 2003. P1-15. Disponible en: http://www2.paho.org/ hq/dmdocuments/2011/eer-amr-costo-infecchospital.pdf (Accedido el 3 de marzo de 2016).

25.- Soria C, Guambo P, Takahashi Y, La Rosa B, Erazo R. Costo de la neumonía asociada a ventilación mecánica en un hospital de cuarto nivel de Guayaquil-Ecuador. Revista Médica de Nuestros Hospitales 2014; 20: 121-3.

26.- Grazioso C, Santay S, Jáuregui E. Costo de las infecciones intrahospitalarias en áreas de cuidado materno-infantil de un hospital de la ciudad de Guatemala. En: González-Salvatierra R. Ed. Costo de la infección nosocomial en nueve países de América Latina. Organización Panamericana de la Salud. Washington D.C.: OPS, 2003. P1-15. Disponible en: http://www2. paho.org/hq/dmdocuments/2011/eer-amr-costo- infecc-hospital.pdf (Accedido el 3 de marzo de 2016).

27.- Mejía C, Villatoro G, de Briz H, García B, Rodas V. Impacto económico de un hospital universitario de la Ciudad de Guatemala. En: González-Salvatierra R. Ed. Costo de la infección nosocomial en nueve países de América Latina. Organización Panamericana de la Salud. Washington D.C.: OPS, 2003. Pl-15. Disponible en: http://www2.paho.org/ hq/dmdocuments/2011/eer-amr-costo-infecchospital.pdf (Accedido el 3 de marzo de 2016).

28.- Villafañe M, Vera A. Costos originados por dos tipos de infección nosocomial en un hospital universitario de Asunción, Paraguay. En: González-Salvatierra R. Ed. Costo de la infección nosocomial en nueve países de América Latina. Organización Panamericana de la Salud. Washington D.C.: OPS, 2003. P1-15. Disponible en: http://www2.paho.org/ hq/dmdocuments/2011/eer-amr-costo-infecchospital.pdf (Accedido el 3 de marzo de 2016).

29.- Suárez E, Casares C, Machuca M. Costo de la neumonía nosocomial y bacteriemia asociada a catéter venoso periférico en un hospital de niños de El Salvador. González-Salvatierra R. Ed. Costo de la infección nosocomial en nueve países de América Latina. Organización Panamericana de la Salud. Washington D.C.:
OPS, 2003. P1-15. Disponible en: http://www2. paho.org/hq/dmdocuments/2011/eer-amr-costoinfecc-hospital.pdf (Accedido el 3 de marzo de 2016).

30.- Vergara T, Fica A. Estudio de costo de las infecciones del torrente sanguíneo asociadas a catéter vascular central en pacientes adultos en Chile. Rev Chilena Infectol 2015; 32: 634-8.

31.- Restrepo M, Anzueto A, Arroliga A, Afessa B, Atkinson M, Ho N, et al. Economic burden of ventilator-associated pneumonia based on total resource utilization. Infect Control Hosp Epidemiol 2010; 31: 509-51.

32.- Vega M, Ávalos D, Holt N, Fretes A. Costo de infecciones asociadas al uso de asistencia respiratoria mecánica y catéter urinario permanente en un hospital del Paraguay. En: González-Salvatierra R. Ed. Costo de la infección nosocomial en nueve países de América Latina. Organización Panamericana de la Salud. Washington D.C.: OPS, 2003. P1-15. Disponible en: http://www2.paho.org/ hq/dmdocuments/2011/eer-amr-costo-infecchospital.pdf (Accedido el 3 de marzo de 2016).

33.- Mathai A S, Phillips A, Kaur P, Isaac R. Incidence and attributable costs of ventilatorassociated pneumonia (VAP) in a tertiary-level intensive care unit (ICU) in northern India. J Infect Public Health 2015; 8: 127-35. 